FORMATION Formation emploi

Revue française de sciences sociales

133 | Janvier-Mars 2016

Heurs et malheurs de l'apprentissage en Suisse

\title{
La formation professionnelle au service de la division sexuelle du travail. L'exemple du métier de la viande en Suisse
}

When professional training serves the sexual division of labour. A case study of the meat sector in Switzerland

Die Berufsbildung im Dienste der geschlechtsspezifischen Arbeitsteilung. Das

Beispiel des Fleischfachberufs in der Schweiz

La formación profesional al servicio de la división sexual del trabajo. El ejemplo de los oficios de la carne en Suiza

Isabelle V. Zinn

\section{OpenEdition}

Journals

Édition électronique

URL : http://journals.openedition.org/formationemploi/4689

DOI : 10.4000/formationemploi.4689

ISSN : 2107-0946

Éditeur

La Documentation française

Édition imprimée

Date de publication : 20 avril 2016

Pagination : 199-214

ISSN : 0759-6340

Référence électronique

Isabelle V. Zinn, « La formation professionnelle au service de la division sexuelle du travail. L'exemple du métier de la viande en Suisse », Formation emploi [En ligne], 133 | Janvier-Mars 2016, mis en ligne le 21 avril 2018, consulté le 30 octobre 2020. URL : http://journals.openedition.org/formationemploi/ 4689 ; DOl : https://doi.org/10.4000/formationemploi.4689 


\title{
La formation professionnelle au service de la division sexuelle du travail
}

\section{L'exemple du métier de la viande en Suisse}

\begin{abstract}
ISABELLE V. ZINN
Sociologue, doctorante et assistante-enseignante à l'université de Lausanne, membre de I'Institut des sciences sociales. Doctorante au Centre d'étude des mouvements sociaux (CEMS) Institut Marcel Mauss (IMM) à l'Ecole des hautes études en sciences sociales (EHESS), Paris. Ses recherches, dans le domaine de la sociologie du travail et des professions, portent sur le "genre en action »
\end{abstract}

Résumé

La formation professionnelle au service de la division sexuelle du travail. L'exemple du métier de la viande en Suisse.

Cette recherche ethnographique sur le métier de la viande, en Suisse, vise à comprendre comment un métier peut être "genré ", et quel est le rôle de la formation professionnelle dans ce processus. Le cursus de la formation professionnelle a récemment été réformé afin de mettre au cœur du métier les tâches considérées comme "féminines " et, ce faisant, de se démarquer de l'image brutale associée à la mise à mort des animaux. Cette division sexuelle du travail cristallise et renforce une bi-catégorisation des sexes et continue à s'organiser à partir de stéréotypes sexués.

Mots clés : enseignement technique-professionnel, apprentissage, division sexuelle du travail, genre, boucher, représentation du travail, Suisse

Abstract

When professional training serves the sexual division of labour. A case study of the meat sector in Switzerland.

This ethnographic study of the meat sector in Switzerland investigates how an occupation becomes gendered, and more particularly the role played by professional training curricula in this labeling process. It shows how the Swiss meat industry has attempted to combat the negative images potentially associated with animal slaughter, notably through the introduction of a new training option, based on so-called " feminine " activities of meat product preparation. However, analysis shows that, rather than promoting women's entry into the profession, this initiative tends to reinforce gender stereotypes and to crystallize a normative sexual division of labour within the occupation. 
Keywords: technical \& vocational education, apprenticeship, gender based division of labour, gender, butcher, perception of work, Switzerland

Journal of Economic Literature: I 21, J 24, J 16, J 44

Traduction : Auteure.

L'existence de métiers « masculins " et d'autres « féminins » ne fait guère question. Cela renvoie à l'investissement différencié des hommes et des femmes dans certains secteurs d'activité (ségrégation horizontale ${ }^{1}$ ), ce qui aboutit à une surreprésentation d'un sexe au sein de certains métiers.

La problématique du genre est entrée dans le champ de la sociologie des professions via l'intérêt pour des questions relatives à la composition sexuée des métiers, à la hiérarchisation sexuée au sein d'un métier donné et à la différenciation sexuée entre métier, à la division sexuelle du travail et aux inégalités qui lui sont liées.

De nombreuses enquêtes se sont intéressées à des métiers dits "masculins ». Elles ont mis en évidence les obstacles auxquels les femmes sont confrontées lorsqu'elles les intègrent, pointant et analysant des mécanismes d'inclusion et d'exclusion (Buscatto, 2003 ; 2008 ; Huppert-Laufer, 1982 ; Kanter, 1977 ; Le Feuvre \& Lapeyre, 2005 ; Mennesson, 2005 ; Pruvost, 2007 ; Zolesio, 2009).

Cet article ${ }^{2}$ est issu d'une recherche ethnographique effectuée au sein du métier ${ }^{3}$ de la viande, en Suisse, qui étudie la manière dont un métier peut être "genré » ${ }^{4}$ D’une part, il analyse comment un métier " masculin » est susceptible de se transformer suite à une réforme de la formation professionnelle visant à un repositionnement du métier articulé autour de tâches considérées comme féminines; d'autre part, il étudie de quelle manière, tout en permettant de recruter plus de femmes, ce processus renforce en définitive une division sexuelle du travail.

Un consensus semble se dégager pour considérer comme étant ségréguée toute profession au sein de laquelle les personnes d'un sexe donné représentent moins de $30 \% 5$ des

1. La notion rend également compte du fait que, même au sein d'un même secteur d'activité, hommes et femmes ne réalisent pas les mêmes activités. La ségrégation verticale se réfere quant à elle à une hiérarchisation au sein d'un métier donné ; elle rend compte de la concentration d'hommes dans les échelons les plus élevés des groupes professionnels.

2. Je remercie les éditeurs-trices de ce numéro, le comité de lecture de la revue Formation Emploi ainsi que Nicky Le Feuvre pour leurs précieux conseils qui portaient sur des versions antérieures de cet article.

3. Nous employons profession et métier de manière interchangeable. Nous utiliserons les termes de métier de la viande ou métier de boucher.

4. Le genre "renvoie à un système social de différenciation (...) qui opère une bicatégorisation relativement arbitraire dans le continuum des caractéristiques sexuelles des êtres humains" (Le Feuvre, 2002, pp.12-13).

5. Il existe plusieurs standards, allant de $10 \%$ à $30 \%$; l'intérêt d'aller jusqu’à $30 \%$ est de montrer que même 
effectifs des professionnels actifs (Heintz, Nadai, Fischer, Ummel, 1995). Le constat qu'un métier est statistiquement sexué constitue, selon nous, une base intéressante pour mener une enquête approfondie visant à identifier comment les individus font l'expérience de cette ségrégation et comment ils y attribuent du sens.

Dans le sillage d'une ethnographie compréhensive qui s'attache à appréhender les actions en situation (Cantelli, Roca I Escoda, Stavo-Debauge, Pattaroni, 2009), cette recherche privilégie l'emprise du genre sur les pratiques, et la manière dont les professionnels s'y confrontent en cherchant à y apporter des solutions ( $c f$. encadré $\mathbf{1}$ ).

Concrètement, cet article vise à comprendre d'une part, comment le fait que les hommes et les femmes n'investissent pas les mêmes activités (la ségrégation horizontale), amorcé et renforcé par le curriculum professionnel, est repris dans les discours ; d'autre part, comment une division du travail apparaît sur les lieux d'exercice de la profession. Dans le cas présent, les organisations professionnelles du métier de la viande s'appuient sur des données chiffrées pour tenter de résoudre des problèmes liés, notamment, à la composition même des professions.

Si le constat statistique joue un rôle important, un processus d'étiquetage renforce considérablement le caractère masculin du métier en question. Quel rôle la formation professionnelle joue-t-elle dans ce processus, et avec quelles conséquences ? Certes, l'évolution de la composition sexuée du métier de la boucherie, dans son ensemble, a été largement amorcée par une restructuration de la formation. Il est moins rare qu'auparavant de rencontrer des femmes exerçant le métier de boucher. Cependant, l'arrivée accrue de femmes dans le métier n'a pas eu comme effet de le rendre plus indifférent au genre. Au contraire, on observe un renforcement de stéréotypes renvoyant soit au féminin, soit au masculin. Ainsi, la réforme de la formation professionnelle a eu comme effet concret et observable le renforcement d'une division du travail basée sur des stéréotypes sexués.

La première partie de l'article présentera quelques caractéristiques essentielles du métier de boucher. On s'intéressera aussi à la manière dont certains membres de la profession agissent sur la structure et l'organisation du métier à travers une réforme de fond du cursus de formation. La seconde partie permettra d'expliciter dans quelle mesure la réforme de la formation professionnelle vise à rendre le métier plus attractif pour les femmes. En conclusion, nous verrons que la réforme de la formation tend in fine à renforcer une bi-catégorisation des sexes en favorisant une division sexuelle du travail.

dans des situations d'avancée en matière de mixité, repli ou reconfiguration du genre demeurent. 


\section{Encadré 1 : Méthodes d'enquête et posture de l'ethnographe}

Notre travail mené sur le métier de la viande, en Suisse, s'inscrit dans une approche qualitative afin de comprendre le sens que confèrent les acteurs à ce qu'ils font. Cet article s'appuie sur une enquête ethnographique, en cours depuis 2011. Elle est menée selon une démarche qui comprend une longue période d'observation participante, avec prise de notes de terrain systématique. La plupart des données ont été recueillies lors de périodes d'observation directes sur divers lieux, tels que des abattoirs, des écoles professionnelles et des boucheries artisanales. Ces observations se sont souvent déroulées lors de stages, d'où une implication et une participation conséquentes aux tâches quotidiennes des enquêté.e.s. Ainsi, nous avons non seulement observé le travail des bouchers, mais également pris part aux activités des enquêté.e.s.

En Suisse, chaque personne désireuse de suivre un apprentissage de boucher (et de tout autre métier exigeant une formation longue) doit effectuer un stage d'une semaine pour connaître les activités qu'implique le métier. Ainsi, les bouchers et bouchères ont l'habitude d'accueillir des personnes inexpérimentées venant se familiariser avec le métier. Cet aspect est très utile dans la mesure où l'ethnographe passe ainsi presque inaperçue lors des stages, et n'est pas $a$ priori considéré comme un intrus. La durée des stages a varié, mais consistait en principe en une semaine dans un même magasin, suivie de deux à trois jours sur place toutes les trois semaines pendant plusieurs mois.

Afin de compléter les observations, des entretiens ont été menés avec divers acteurs du métier (bouchers, patrons de boucheries artisanales, professeurs d'écoles professionnelles, personnes actives au sein d'associations professionnelles, ainsi que des apprenti-e.s). Ces entretiens semidirectifs ont été menés à l'aide d'une grille d'entretien et ont duré en moyenne 90 minutes. Si les thèmes traités lors des entretiens ont varié suivant les interlocuteurs, ils se structuraient autour de quatre grands axes : le parcours professionnel, la formation professionnelle, les activités professionnelles ainsi que des questions relevant de la composition sexuée du métier, sans exclure d'autres thématiques soulevées par la dynamique de l'entretien. Les entretiens ont ensuite été retranscrits intégralement et l'on s'est alors attaché à dégager des thèmes transversaux et des idées clés permettant de mener une analyse thématique et comparative.

Il va sans dire que les observations in situ ont donné lieu à des discussions informelles, qui ont enrichi et éclairé nettement le matériau empirique recueilli grâce aux entretiens semi-directifs.

Un certain nombre de documents professionnels, tel que des rapports annuels, les curricula de formation et du matériel promotionnel sont également pris en compte au cours de l'analyse.

\section{Réformer la profession pour transformer ses membres}

Le métier de boucher nécessite une formation prolongée (CFC de boucher-charcutier ${ }^{6}$ ), comporte des aspects commerciaux, relationnels et techniques, ainsi qu'une structure pro-

6. Le Certificat fédéral de capacité (CFC) est un diplôme reconnu au niveau suisse et délivré par les pouvoirs publics. Il s'obtient généralement autour de l'âge de 19 ans, après une formation professionnelle duale (CSFO, 2012) d'une durée de trois ou quatre ans selon les cas. 
fessionnelle (un cursus de formation formalisé, entreprises indépendantes) qui permet aux individus de devenir indépendants. Par ailleurs, le métier est caractérisé par de longues journées de travail et des "petits salaires", comme le confirment souvent les bouchers euxmêmes et les chiffres officiels (OFS, 2008). Par ailleurs, il s'agit d'un corps de métier autoorganisé, composé d'associations et des unions professionnelles faîtières ${ }^{7}$.

Selon les derniers chiffres officiels disponibles (OFS, 2011), en Suisse, au sein du métier de boucher, la répartition selon le sexe est la suivante : 777 femmes pour 7867 hommes, soit un taux de féminisation de $9 \%$, taux qui a légèrement augmenté au cours des quarante dernières années.

Statistiquement masculine, la profession a connu un déclin au cours des dernières décennies, le nombre de bouchers ayant été divisé par deux en quarante ans (OFS, 2011).

Ce déclin numérique est appréhendé par les organes faittiers de la profession comme un manque de relève et comme un problème de recrutement, déstabilisant la transmission du savoir au sein du métier et, à terme, mettant en péril la profession. Dans les différentes boucheries ayant fait l'objet d'observation, les bouchers déclarent de manière récurrente qu'ils peinent à trouver des apprentis, en particulier de "bons apprentis ", c'est-à-dire des jeunes à la fois "motivés» et " habiles». Dans certains cas, les employeurs n'ont pas recruté dans les délais et n'ont ainsi pas pu pourvoir un poste vacant ou une place d'apprentissage ; dans d'autres cas, ils ont recruté des apprentis en "gestion de commerce ", amenés à se spécialiser dans le domaine de la vente en boucherie. Si ce dernier cas de figure tend à pallier le manque de main-d'œuvre pour le magasin en question, il va sans dire qu'il ne représente en aucun cas, pour les patrons bouchers et le métier lui-même, une solution satisfaisante permettant de conserver et développer le savoir-faire propre au métier de la viande.

Face à ce manque d'effectif, des artisans bouchers membres d'organisations faîtières, c'està-dire des professionnels du métier, ont mené une réflexion. Ils se sont en particulier intéressés à l'image sociale du métier, associée à la mise à mort et au sang et qui, constatent-ils, le connote négativement. Lors de plusieurs rencontres avec des bouchers, l'image sociale, qui serait associée à la "cruauté ", a suscité des débats et a été présentée comme facteur explicatif du déclin du métier. Il conviendrait dès lors de recruter davantage de personnes afin de permettre à la profession de survivre, et ce grâce à une restructuration du cursus de formation. Une commission dédiée et des groupes cibles ont été mis en place et ont proposé des mesures de restructuration concrètes ; au printemps 2006, les délégués de la branche carnée ont accepté le projet de réforme, qui a ensuite été soumis à l'Office fédéral de la formation professionnelle et de la technologie ${ }^{8}$.

7. Il s'agit d'une expression suisse désignant le comité directeur de l'interprofession.

8. L'OFFT, devenu par la suite le Secrétariat d'Etat à la formation, à la recherche et à l'innovation (SEFRI). 
Des spécialistes externes à la branche (notamment des responsables des départements de l'éducation des cantons) ont alors examiné le projet et ont donné leur aval à cette réforme, qui est entrée en vigueur au $1^{\text {er }}$ janvier 2008 (USPV, rapport annuel 2006, p. 22). Auparavant, il n'était pas absolument impossible, pour un apprenti, de devenir boucher sans passer par l'apprentissage de l'abattage du bétail. A présent, ce cas de figure est reconnu comme pouvant faire l'objet d'un choix de plein droit. Autrement dit, devenir boucher sans passer par l'acte de la mise à mort est aujourd'hui chose acquise. ${ }^{9}$

Dès lors, la formation comprend quatre sous-spécialisations ${ }^{10}:$ " production " (en premier lieu, abattage du bétail ainsi qu'en partie désossage-découpage.), "transformation ", " transformation industrielle " (préparation de produits prêts à la vente, saucisses et charcuterie) et "commercialisation " (préparation de produits traiteur, de plats froids, de plats prêts à cuisiner, service et conseils à la clientèle) (Présentation de la formation, UPSV ${ }^{11}$ ).

Les apprentis choisissent aujourd'hui leur spécialisation au moment où ils entrent en apprentissage. Ce choix étant médiatisé par les besoins des commerces et de l'industrie, l'apprenti-e sera amené-e à choisir plutôt l'une ou l'autre spécialisation suivant les besoins des professionnel.le.s formant des apprenti.e.s.

La spécialisation de la formation se traduit également dans les activités sur les lieux de travail, où les bouchers sont en principe cantonnés à leur spécialisation et aux tâches qui y sont inhérentes : la personne dont l'activité principale est la vente des produits ne désossera pas de grandes pièces et ne participera pas à l'abattage. Cette spécialisation se double d'ailleurs d'une spatialisation des activités; certains locaux sont en effet spécifiques à l'une ou à l'autre des tâches (le "hall d'abattage " pour la mise à mort ; le "laboratoire " pour le découpage et le désossage ; le "magasin " pour la commercialisation des produits carnés).

Il existe d'ailleurs une proposition plus récente qui semble s'inscrire dans la stratégie qui vient d'être présentée : créer une cinquième filière, voire, comme on peut le lire dans les rapports annuels de l'Union professionnelle suisse de la viande (UPSV), un " nouveau métier ». Si en 2010, l'intitulé proposé pour cette nouvelle branche était "Spécialiste Gourmet » (rapport annuel 2010, p. 13), en 2011, a été proposée celle de "spécialiste de produits frais». Lidée restait la même : présenter les boucheries avant tout comme des lieux de commercialisation de produits carnés toujours plus raffinés et prêts à consommer, en

9. Il serait intéressant de mieux analyser le rôle que joue potentiellement la réglementation en matière d'hygiène et de sécurité alimentaire dans la restructuration de la formation, mais par manque de place, ceci ne sera pas abordé dans le présent article.

10. Ces quatre spécialisations, sauf celle de la transformation industrielle, ne renvoient pas nécessairement à des types d'entreprises distinctes. Même si de moins en moins de boucheries artisanales pratiquent l'abattage du bétail, il n'est pas exclu que dans une même boucherie se retrouvent des personnes issues de chacune des spécialisations. Néanmoins, on observe une certaine division du travail entre les entreprises : dans les abattoirs, il est rare de trouver des personnes ayant suivi la filière " commercialisation ".

11. http://www.carnasuisse.ch/fr/formation/boucher-charcutier-documents.php 
se démarquant ainsi de la "cruauté " que peut représenter l'abattage. Autrement dit, le discours professionnel tente de dissocier la viande de l'animal ${ }^{12}$.

"Depuis quelques années déjà, l'économie carnée suisse constate qu'elle manque de spécialistes compétents pour des secteurs entiers. C'est ainsi que les entreprises artisanales de la boucheriecharcuterie ont fortement développé le secteur de l'offre de repas prêts et autres, du catering [service traiteur] aux menus pré-cuisinés, et jusqu'à l'organisation d'events [événements]. Il s'avère toujours plus souvent que le concept de formation proposé aujourd'hui (...) ne parvient pas à couvrir les compétences spécifiques pour ces domaines. C'est pourquoi l'UPSV recherche des solutions qui permettent de combler cette lacune (...) : la création d'un nouveau métier est en préparation avec les branches qui nous sont proches ${ }^{13}$. Pour le moment, le nom choisi est celui de "Spécialiste en produits frais". Le point fort de ce nouveau métier serait une formation qui mettrait l'accent sur les capacités manuelles dans la préparation et la transformation de produits convenience [plats cuisinés] et de produits frais, ainsi que les activités purement de vente, (...) et des services de livraison à domicile et service à la clientèle. " (UPSV, rapport annuel 2011, p. 13).

L’objectif est sans doute de véhiculer une image plus lisse du métier en proposant davantage de produits prêts à consommer ; en effet, au cours des quinze dernières années, en Suisse, la consommation de viande est passée de $53.2 \mathrm{~kg} /$ par habitant en 1999 à $50.5 \mathrm{~kg}$ en 2013 (OFS, bilan de la viande, 2014). En ce sens, il s'agit également d'enrayer cette baisse en proposant une diversification grâce aux produits transformés qui correspondent de plus en plus aux attentes des consommateurs et consommatrices.

Dans les discours des bouchers rencontrés, un autre facteur est évoqué, celui de la concurrence des grandes surfaces. D'un côté, les bouchers souhaitent se démarquer des rayons "boucherie-charcuterie » des grandes surfaces en proposant un conseil personnalisé à leurs client.e.s; de l'autre, pour faire face à cette concurrence, ils sont amenés à s'aligner sur la même stratégie d'offre de produits prêts à consommer.

Dans la suite de l'article, nous nous intéresserons à l'idéal du boucher ainsi qu'au " nouveau » boucher, devenu le spécialiste de la viande, pour ensuite aborder la création de filières de formation "féminines ». Cela permettra de comprendre la manière dont l'interprofession souhaite revaloriser le métier en accueillant davantage de femmes.

12. Nous observons un discours similaire en France où la consommation de viande n'a cessé de baisser ces dix dernières années (http://www.la-viande.fr/nutrition-sante/niveaux-consommation-viande-France, consulté le 25 juillet 2014), ce qui a contraint les boucheries à innover : "le boucher est devenu un cuisinier à part entière " remarque un des invités dans cette émission où il s'agit de découvrir le métier de boucher (http:// www.lcp.fr/emissions/j-aimerais-vous-y-voir/vod/159351-catherine-quere-deputee-socialiste-de-charentemaritime-bouchere). Les responsables de la boucherie en question précisent que la partie traiteur représente désormais $60 \%$ du chiffre d'affaires.

13. Nous pouvons supposer qu'il s'agit des métiers de bouche tels que les métiers de la restauration et de l'agroalimentaire. 


\subsection{Entre spécialisation et polyvalence}

Si la spécialisation de la formation professionnelle est mise en place par les associations professionnelles, certains bouchers rencontrés ne sont pas satisfaits de ce nouveau développement : "Je ne comprends pas pourquoi ils veulent toujours et encore plus spécialiser, c'est une tendance, comme chez les médecins. On n'aura plus que des spécialistes, et aucun généraliste. C'est n’importe quoi!» (Hervé, boucher diplômé, 32 ans, travaille dans une boucherie artisanale de campagne).

Ce manque d'enthousiasme est présent chez un autre boucher, patron d'une boucherie artisanale de grande taille. Il insiste sur le fait que, chez lui, les apprentis font de tout sauf l'abattage - qu'il n'impose pas à ses apprentis n'exprimant pas de souhait particulier d'y assister et de s'y former. Sinon, dit-il : "ils vont passer du magasin au laboratoire et autant vendre les produits que de faire du désossage!" (boucher diplômé, 40 ans, patron d'une boucherie artisanale de campagne). Le patron insiste sur le fait qu'il a besoin de personnes sachant tout faire, ceci tant pour des raisons de rentabilité que d'organisation du travail.

Ce cas de figure est intéressant : les objectifs de la formation professionnelle réformée, à savoir proposer une spécialisation de plus en plus poussée, ne coïncident pas forcément avec les besoins des patrons de boucheries artisanales; pour ces derniers, le bon boucher reste celui qui est en mesure de réaliser l'ensemble des activités relevant du métier, et dans leur vision des choses, le travail bien fait se juge à l'aune d'une polyvalence des professionnels, et non d'une spécialisation sur un nombre de gestes limités. Ce discours corrobore celui d'un autre patron boucher, qui estime qu'il a de la chance car, dans sa boucherie, on " travaille encore du début à la fin, on fait tout, depuis l'achat du bétail jusqu'à la vente, donc on a le processus de toute la chaîne alimentaire " (59 ans, boucher diplômé, patron d'une boucherie artisanale d'une ville de petite taille).

Ce témoignage révèle que ce boucher accorde une grande importance au fait de pouvoir " tout faire" pour bien faire son métier. Si être un bon boucher n'exclut pas que l'on soit également bon vendeur, le bon professionnel doit impérativement maîtriser toute la chaîne de production et ne peut pas se contenter de la commercialisation des marchandises. Nous verrons que cet idéal de «bon boucher " n'est pas neutre du point de vue du genre, mais qu'il relève, au contraire, d'une figure masculine.

\subsection{Le « nouveau » boucher ou le spécialiste de la viande}

En Suisse alémanique, ${ }^{14}$ en 2008 (Rapport annuel 2011, p. 13), soit en même temps que la restructuration des cursus de formation, la profession a également changé sa propre appellation et celle de ses employés : le métier est devenu le Fleischfachberuf (pourrait être

14. En Suisse romande, la profession a conservé son nom. 
traduit par "métier de la viande ») ; le boucher, traditionnellement le Metzger, est désormais le Fleischfachmann, le spécialiste de la viande.

Dans les entretiens conduits avec des professionnels et des représentants de la formation professionnelle, ce changement de nom est revendiqué comme le souhait de modifier l'image de la profession, en faisant ressortir l' " expertise " et la "spécialisation " et en se débarrassant ainsi d'une appellation qui est considérée comme directement liée à l'abattage des bêtes. Autrement dit, il s'agit d'occulter les activités liées à la mise à mort, qui demeure malgré tout essentielle à toute pratique du métier. Une affiche publiée sur le site "Swiss Meat People " ${ }^{15}$, pour promouvoir le métier et qui devrait plus particulièrement permettre de recruter davantage d'apprenti-e-s, comme l'indique le slogan Schliesse dich dem Team an ("rejoins l'équipe»), illustre cette volonté de la profession :

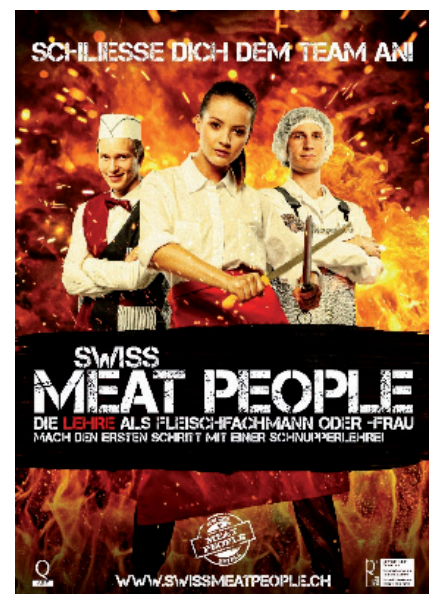

Au premier plan de l'image figure une jeune femme en train de manipuler deux fusils à aiguiser, et la mise en scène globale évoque une action héroïque faisant davantage penser à la promotion d'un film d'action qu'au métier de la viande. Cet aspect héroïque soustend aussi les propos des enquêtés, comme par exemple lorsqu'un boucher diplômé, 42 ans, responsable dans un abattoir industriel, mentionne une des qualités requises pour être un "bon boucher ", à savoir la fierté de faire ce métier, "car qui d'autre le ferait?".

Notons aussi le recours à la langue anglaise (sur cette affiche ainsi que dans l'extrait du rapport annuel de la UPSV cité ci-dessus - convenience, events, catering). Si l'emploi d'anglicismes est habituel dans la partie germanophone de la Suisse, ce choix semble

15. Mot à mot, "Swiss Meat People » signifie " les suisses de la viande », renvoyant aux bouchers suisses. Ce site, initié par l'Union professionnelle suisse de la viande, n'existe qu'en allemand et regroupe des informations importantes liées à la formation, au championnat suisse des bouchers-charcutiers, aux parcours professionnels et recense et annonce des événements de la branche (http://swissmeatpeople.ch/index.asp). 
avoir pour but de véhiculer une image plus " cool " et " ouverte " du métier et ainsi d'attirer les jeunes gens ${ }^{16}$.

\section{Créer des filières de formation «féminines »}

Cette manière d'appréhender les problèmes auxquels la profession est confrontée révèle que les acteurs impliqués dans l'organisation de la profession se réfèrent eux-mêmes à la composition sexuée de la profession.

En promouvant les activités de "transformation » et de " commercialisation » de la viande, les acteurs semblent vouloir occulter la mise à mort des animaux qui, aux yeux de certains, relève de la "cruauté » et limite l'attrait de la profession. En outre, toutes les activités qui relèvent explicitement du travail physique, notamment les activités liées à l'abattage du bétail et au désossage des carcasses, sont reléguées au deuxième plan, au profit d'autres activités, comme en témoignent notamment les propos qu'un professeur d'une école professionnelle tient devant ses élèves : "L'avenir du métier, c'est le conseil... vous ne pouvez pas venir avec un tablier plein de sang et ne rien connaître à la cuisine. Traçabilité et conseil! Il faut jouer là-dessus, vous devenez des conseillers! »(CFC de cuisiner et de boucher-charcutier, 39 ans, professeur dans une école professionnelle).

De l'enquête ethnographique, il ressort que le fait de rendre les conditions d'exercice " plus propres " vise à attirer un nouveau public, plus particulièrement féminin. En consultant les chiffres de l'OFS, repris par l'USPV, le pari semble être gagné : entre 2007 et 2008 (période de mise en œuvre de la restructuration du cursus de formation), le nombre d'apprenties filles a augmenté de 36 à 44 sur un total de 310 apprenti.e.s, pour atteindre 49 apprenties (sur 254) en 2013. Une nette majorité de ces apprenties ont choisi la filière " commercialisation" (USPV, rapports annuels 2006-2013). Ce public serait plus apte à la commercialisation des marchandises qu'au travail physique en abattoir ${ }^{17}$. Les femmes

16. A ce propos, mentionnons un article paru en juillet 2014 dans le "Tages-Anzeiger ", relatant que plus de 8000 places d'apprentissage en Suisse - sur environ 80000 (http://www.sbfi.admin.ch/aktuell/medien/00483/00594/index.html?lang=fr\&msg-id=53295) - n'ont pas encore été pourvues et que plusieurs branches (dont celle de la viande) essaieraient d'attirer des jeunes avec des images et slogans attractifs et vivants ("Jeder Metzger ein Spitzensportler" : http://www.tagesanzeiger.ch/wirtschaft/unternehmen-undkonjunktur/Jeder-Metzger-ein-Spitzensportler/story/22563898, consulté le 29 juillet 2014). Il semble qu'en Allemagne le métier de la viande soit confronté à un problème similaire : il manque de candidat-e-s et les associations professionnelles essaient de revaloriser l'image du métier afin de recruter davantage de personnel ("Jung-Metzger-Wettbewerbe sollen Fleischer-Image aufpolieren", http://www.sat1 regional.de/panorama-video/article/metzger-wettbewerbe-sollen-fleischer-image-aufpolieren-156393.html, consulté le 16 novembre 2014).

17. Contrairement à ce que l'on pourrait penser, mais sans vouloir minimiser l'aspect physique effectif du travail à accomplir, mes observations dans différents dispositifs d'abattage révèlent que grâce à la mécanisa- 
contribueraient à la revalorisation du statut du métier en rompant avec l'image " rustre " de la boucherie.

La promotion même de la profession passe alors par l'accueil de plus de femmes au sein du métier. Autrement dit, le fait d'attirer et de recruter des femmes devrait non seulement combler le déficit de main-d'œuvre, mais également revaloriser l'image sociale du métier. La réforme est ainsi motivée par les difficultés que rencontre le métier de la viande : l'orientation vers des produits prêts à consommer se reflète dans les nouvelles spécialisations proposées et permet en même temps d'attirer plus de femmes en proposant des « filières féminines ". Il semble ainsi que le souhait d'ouverture aux femmes représente un facteur déterminant des changements opérés.

Les bouchères rencontrées - sauf une, spécialisée dans la vente et la préparation des produits carnés - reprennent ce même type de raisonnement, en expliquant que la nouvelle option proposée dans le cursus de formation leur plairait bien, car cela permettrait d'être bouchères sans abattre de bêtes, et ainsi d'échapper à des conditions d'exercice physiquement plus rudes (Perrot, Zinn, 2015).

De ce point de vue, la rencontre avec le directeur des achats de bétail, de l'abattage et de la formation d'une structure industrielle a été révélatrice. La discussion a tout d'abord porté sur le métier de boucher en général et ses conditions d'exercice ; ensuite, la conversation s'est portée sur la composition sexuée du métier : "oui... je ne vois aucun problème de fond si les dames viennent dans le métier, mais il faut savoir qu'il s'agit d'un métier physique, quion travaille dans le froid, qu'on fait beaucoup d'heures. Il est vrai, dans une boucherie où il faut encore porter les carcasses, c'est difficile, mais les dames peuvent faire autre chose, elles peuvent très bien intégrer la filière "commercialisation". Mais encore, il faut savoir que leur morphologie va changer, elles auront moins les épaules fines. C'est vrai, ici dans l'abattage, on a peu de dames, mais il y en a... pas aux postes difficiles quand-même."

Les professionnels semblent tenir pour acquis que les compétences supposées naturelles des femmes, associées à la "propreté " et à la "douceur", seraient bénéfiques à la profession. Cette conception entraîne non seulement une stéréotypisation des compétences, mais rend également les activités genrées. Par conséquent, le peu de femmes bouchères sont dès lors directement associées à des activités telles que la vente des produits carnés en magasin ou le parage fin des pièces bouchères, et non à l'abattage du bétail et au désossage de carcasses.

Dans ce sens, il est intéressant de noter que le directeur des achats évoqué ci-dessus ne s'oppose pas à l'entrée des femmes dans le métier, mais leur accorde, d'emblée, une place

tion des procédés de production et à un modèle industriel du travail, le processus d'abattage n'exige pas un investissement physique trop rude de la part des individus. Autrement dit, si les conditions d'exercice ne sont certes pas faciles (travail dans le froid, nombre d'heures par semaine élevé, des "petits salaires ", etc.), le travail physique rude ne semble pas primer sur les autres activités. 
spécifique au sein du métier et justifie son avis par le caractère physique de certains travaux à effectuer. Autrement dit, bien que les femmes soient plus faibles, elles sont néanmoins bienvenues, mais devront rester à la place qui leur est attribuée.

Si la restructuration de la formation et l'entrée des femmes dans le métier visent une mixité statistique, cela ne s'accompagne pas d'une uniformisation des compétences. On constate donc une symbolique des tâches et des gestes qui sont associés de manière différenciée selon les sexes. Si une telle distribution sexuée est problématique, supposant une bi-catégorisation des sexes, elle semble en même temps permettre aux femmes de déléguer un travail jugé pénible.

En effet, toute la partie du métier liée à la mise à mort des animaux est considérée par l'ensemble des personnes rencontrées comme ayant un côté " repoussant » et "cruel", qu'il s'agit de «cacher» au grand public. ${ }^{18}$ Des travaux plus «nobles» et moins pénibles (Gallioz, 2007), comme le parage fin de morceaux et la commercialisation des produits carnés, sont globalement confiés aux femmes. ${ }^{19}$ Ceci établit un lien entre une catégorie de genre, une activité de travail, et des qualités soit spécifiquement " masculines ", soit "féminines ». Il est intéressant de voir comment les catégorisations de genre se manifestent et comment la ségrégation horizontale renforcée par le nouveau curriculum se traduit sur le terrain par une légitimation sociale de la division sexuelle du travail.

La modification du cursus de formation devrait amorcer une féminisation du métier ; cependant, en réservant aux femmes une place particulière, elle renforce en même temps des stéréotypes sexués. C'est ainsi que la formation professionnelle favorise une organisation du travail basée sur une division sexuelle.

Ces exemples illustrent la façon dont les instances représentatives d'une profession, et par la suite ses membres ordinaires, tentent d'agir sur l'image publique de la boucherie et sur la composition sexuée de ses effectifs. Ce processus peut être retracé et schématisé comme tel : « la profession » a fait l'expérience d'un déficit de personnel (constat) et en a conclu que son image sociale n'était pas bonne (diagnostic); elle estime que cette mauvaise image est liée à certaines activités professionnelles et aux qualités imputées à ceux qui les mettent

18. Selon C. Rémy (2004), cet acte peut conférer à celui qui tue un statut de dominant au sein du groupe. En ce sens, le fait d'associer le travail dans l'abattoir au "sale boulot " (Hughes, 1996 [1971]) n'est pas un geste anodin. Mais précisons que le «sale boulot » ne rime pas forcément avec la dévalorisation de la personne qui l'exécute et peut même lui conférer un certain prestige au sein de la communauté.

19. Il convient néanmoins de distinguer deux niveaux : les objectifs de la réforme et du système de formation professionnelle, à savoir casser l'image brutale du métier par une féminisation des effectifs, d'une part, le niveau de la pratique, où l'on peut observer une tendance de ce que C. Marry (2004) a appelé les «bastions virils ", donc une certaine perpétuation des stéréotypes sexués, d'autre part. Certains bouchers semblent mettre en œuvre des stratégies afin de mieux supporter leur travail et se livrent ainsi, sur le mode de la plaisanterie, à des remarques sexuées et sexistes au travail. 
en œuvre (analyse) et propose de modifier sa composition sexuée en recrutant plus de femmes (moyen et solution).

Une arrivée accrue de femmes, voulue et promue par les représentants institutionnels, vise alors, dans ce cas, et contrairement au prisme associant féminisation à déclassement (Reskin et Roos, 1990 ; Riska et Weger, 1993 ; Bourdieu, 1979), à rendre la profession plus " acceptable » pour le grand public, voire à une revalorisation sociale (CacouaultBitaud, 2001 ; Le Feuvre, 2003). Il semble par ailleurs que l'association entre déclassement social et féminisation vaut plus souvent pour les professions dites prestigieuses que pour les métiers qui sont socialement dévalorisés et où l'entrée des femmes est potentiellement un signe de revalorisation.

\section{Conclusion}

Lanalyse développée ici visait à mieux comprendre le rôle de la formation professionnelle sur le renforcement du caractère sexué de l'activité de boucher et la division sexuelle du travail.

La profession s'est récemment restructurée et la formation professionnelle comporte aujourd'hui quatre filières distinctes. Cette transformation vise à enrayer le déclin de la profession en la rendant plus attractive pour un public plus large, en particulier féminin. En effet, les statistiques portant sur la répartition selon le sexe, au sein du métier, ont permis aux responsables de la profession d'identifier le public féminin comme le vivier de main-d'œuvre le plus prometteur ; d'où l'importance de mettre en avant la composition sexuée de la profession, pour pouvoir s'y appuyer.

Les modifications opérées sur les cursus de formation ont engendré un travail de promotion spécifique : permettre aux professionnel.le.s d'intégrer pleinement le métier sans nécessairement passer par l'abattage des animaux, élément qui a été identifié comme l'obstacle principal au recrutement d'un effectif suffisant.

Si le métier reste marqué par une très forte présence masculine, cette situation est susceptible d'évoluer. Dès lors, les tentatives pour attirer plus de femmes passent par un effort de présentation et de représentation du métier visant une mise à distance de la "cruauté » et des aspects les plus physiques du métier.

Notre enquête sur le métier de la viande est particulièrement intéressante puisque dans ce métier, la virilité traditionnelle semble être mise à mal. Ce n'est pas le cas d'autres enquêtes qui ont montré, par exemple dans le cas de la police (Pruvost, 2007), que la féminisation du métier ne modifiait pas la valorisation des normes masculines; ou dans celui plus large du compagnonnage (Adell, 2011), où l'entrée des femmes permet aux hommes de se libérer de tâches n'étant pas au cour du métier (considérées comme " moins masculines ") ; ou encore dans les métiers du bâtiment, où les femmes, considérées comme "improductives»(Gallioz, 2006, p. 5), sont confinées dans les bureaux. 
La restructuration de la formation professionnelle vise à mettre au cœur de la profession des " tâches féminines » et à rompre ainsi avec une image brutale associée à la mise à mort des animaux. Autrement dit, les tâches qui renvoient à une valorisation positive et celles qui sont présentées au grand public sont également celles qui sont plutôt exercées par les femmes. Néanmoins, si la réorganisation du métier via la modification du cursus de formation doit permettre, sur le long terme, de recruter davantage de femmes et d'amorcer ainsi une féminisation du métier et par la suite une plus grande équité statistique, elle tend aussi à renforcer des stéréotypes sexués en associant femmes et hommes à des types d'activités distinctes. Autrement dit, la division du travail s'organise toujours à l'aune de stéréotypes sexués. Se pose également la question du sens exact de la volonté d'ouverture aux femmes : la restructuration de la formation vise-t-elle réellement une mixité ou s'agit-il, en premier lieu, d'une stratégie pour assurer la transmission du savoir professionnel qui passe par le recrutement de davantage de main-d'œuvre, en l'occurrence féminine?

Enfin, ce qui pourrait tout d'abord apparaitre pour les femmes, comme une délégation du " sale boulot ", est en réalité plus complexe : valoriser la spécialisation de la formation, et en particulier proposer désormais des spécialisations adéquates aux femmes - des « filières féminines " - est au cœur des discours adressés par les associations professionnelles au public, en particulier externe à la profession. Cette vision est cependant contestée par plusieurs bouchers rencontrés ; ils persistent en effet à identifier le bon boucher à un professionnel polyvalent, maîtrisant toute la chaîne de production. Et ce professionnel, loin d'être neutre du point de vue du genre, constitue une figure masculine. Autrement dit, en associant aux femmes le "travail propre ", au sens littéral, on vend du "sale boulot " à ces dernières, car au sein de la profession, il vaut encore mieux être généraliste que spécialiste.

Si la réforme de la formation professionnelle est susceptible de transformer le métier en mettant en son cour des tâches considérées comme féminines, le présent cas montre qu'une féminisation amorcée renforce la ségrégation horizontale et que les hommes et les femmes n'effectuent pas les mêmes activités au sein d'un même métier.

\section{Bibliographie}

Adell N. (2011), "Le Genre en faces », Revue européenne des sciences sociales, vol. 49, n² pp. 231-257.

Bourdieu P. (1979), La distinction. Critique sociale du jugement, Paris, Les Editions de Minuit.

Buscatto M. (2003), "Chanteuse de jazz n’est point métier d'homme ", Revue française de sociologie, vol. $44, \mathrm{n}^{\circ} 1$, pp. 35-62.

Buscatto (2008), "Tenter, rentrer, rester : les trois défis des femmes instrumentistes de jazz ", Travail, genre et sociétés, vol. 1, n 19, pp. 87-108. 
Cacouault-Bitaud M. (2001), "La feminisation d'une profession est-elle le signe d'une baisse de prestige ?", Travail, genre et sociétés, vol. 1, n 5, pp. 91-115.

Cantelli F., Roca I Escoda M., Stavo-Debauge J. et Pattaroni L. (2009), Sensibilités pragmatiques. Enquêter sur l'action publique, Bruxelles, Bern, Berlin, Frankfurt am Main, New York, Oxford, Wien, Peter Lang.

Centre suisse de services (2012), Formation professionnelle, orientation professionnelle, universitaire et de carrière (CSFO), CFC sans apprentissage (article 32), http://www.berufsberatung.ch/dyn/10641.aspx, consulté le 30 juin 2012.

Gallioz S. (2006), "Force physique et féminisation des métiers du bâtiment ", Travail, genre et sociétés, vol. 2, n 16, pp. 97-97.

Gallioz S. (2007), "La féminisation des entreprises du bâtiment : le jeu paradoxal des stéréotypes de sexe ", Sociologies pratiques, vol. 1, n 14, pp. 31-44.

Heintz B., Nadai E., Fischer R. et Ummel H. (1995), Ungleich unter Gleichen, Frankfurt, New York Campus Verlag.

Hughes E.C., (1996 (1971)), Le regard sociologique, Paris, Editons de l'Ecoles des Hautes Etudes en Sciences Sociales.

Huppert-Laufer J. (1982), La féminité neutralisée? Les femmes cadres dans l'entreprise, Paris, Flammarion.

Kanter R. M. (1977), Men and Women of the Corporation, New York, Basic Books.

Le Feuvre N. (2002), Le genre : de la catégorisation des sexes, Paris, Numéro spécial de UTINAM : Revue de sociologie et d'anthropologie.

Le Feuvre N. (2003), Penser la dynamique du genre : parcours de recherche, dossier pour l'habilitation à diriger des recherches, université de Toulouse Le Mirail, Toulouse.

Le Feuvre N., Lapeyre N. (2005), « Féminisation du corps médicale et dynamiques professionnelles dans le champ de la santé ", Revue française des Affaires sociales, vol. 1, n¹, pp. 59-81.

Marry C. (2004), Les femmes ingénieurs. Une révolution respectueuse, Paris, Belin.

Mennesson C. (2005), «Les femmes guides de haute montagne : modes d'engagement et rapports au métier ", Travail, genre et sociétés, vol. 1, n 13, pp. 117-137.

Office fédéral de la statistique (OFS) (2014), Bilan de la viande 1999-2013, http://www. bfs.admin.ch/bfs/portal/fr/index/themen/07/03/blank/data/01/04.html.

OFS (2011), Erwerbstätige nach Geschlecht sowie beruflicher Tätigkeit und höchster abgeschlossener Ausbildung (aggregiert) sowie Fünfahresaltersklassen, 1970-2000 [Personnes actives occupées selon le sexe, l'activité professionnelles et le plus haut diplôme obtenu (données agrégées), par classes d'âge de cinq ans, 1970-2000]. Neuchâtel. 
OFS (2008), L'enquête suisse sur la structure des salaires, Neuchâtel.

Perrot A., Zinn I. V. (2015), « Du tâtonnement ethnographique au discernement de sens: enquêtes participatives en boucherie et dans la zone d'attente des mineurs isolés étrangers ", Recherches inductives, vol. 2, n 2, pp. 129-154.

Pruvost G. (2007), « La dynamique des professions à l'épreuve de la féminisation : l'ascension atypique des femmes commissaires ", Sociologie du travail, vol. 49, nº 1, pp. 84-99.

Rémy C. (2004), "L'espace de la mise à mort de l'animal. Ethnographie d'un abattoir ", Espaces et sociétés, vol. 3, n 118, pp. 223-249.

Reskin B.F., Roos P. A. (1990), Job Queues, Gender Queues. Explaining Women's Inroads into Male Occupations, Philadelphie, Temple University Press.

Riska E. \& Weger K. (1993), Gender, Work and Medecine. Women and the Medical Division of Labour, Londres, Sage.

Swiss Meat People, Union Professionnelle de la Viande Suisse (UPSV), http://www. swissmeatpeople.ch/, consulté le 22 juillet 2014

Union Professionnelle de la Viande Suisse (UPSV), Rapports annuels, 2006-2011, http:// www.metzgerei.ch/fr/association/rapports-annuels.php, consulté le 16 juillet 2014.

Zolesio E. (2009), Des femmes dans un métier d'hommes : L'apprentissage de la chirurgie ", Travail, genre et sociétés, vol. 2, n 22, pp. 117-133. 